\title{
Khinchin's theorem in $k$ dimensions with prime numerator and denominator
}

\author{
by \\ Huw Jones (Cardiff)
}

1. Introduction. This paper is devoted to the proof of the following result.

THEOREM. Let $\psi_{1}(n), \ldots, \psi_{k}(n)$ be non-increasing, positive functions of a positive integer variable $n$. Then the simultaneous inequalities given by:

$$
\left|\alpha_{i}-\frac{q_{i}}{p}\right|<\frac{\psi_{i}(p)}{p}
$$

for $1 \leq i \leq k$ have infinitely many or finitely many solutions in primes $p, q_{i}$ for almost all $\boldsymbol{\alpha}=\left(\alpha_{1}, \ldots, \alpha_{k}\right)$ in $\mathbb{R}^{k}$, according to whether the sum

$$
\sum_{n=2}^{\infty} \frac{\psi_{1}(n) \ldots \psi_{k}(n)}{(\log n)^{k+1}}
$$

diverges or converges respectively.

In the English translation of "Continued Fractions" [7] by A. Ya. Khinchin the theorem to which the title refers is Theorem 32 on page 69. It can be stated as follows:

Suppose that $\psi(x)$ is a positive continuous function of a positive variable $x$ and that $x \psi(x)$ is a non-increasing function. Then the inequality

$$
\left|\alpha-\frac{m}{n}\right|<\frac{\psi(n)}{n}
$$

has, for almost all $\alpha$, an infinite number of solutions in integers $m$ and $n$ (with $n>0)$ if $\sum_{n=1}^{\infty} \psi(n)$ diverges. If on the other hand the sum converges then for almost all $\alpha$ there are only a finite number of solutions to the inequality.

This elegant theorem was proved by Khinchin in the context of continued fractions and using results of Borel and Bernstein. It has been the subject

2000 Mathematics Subject Classification: Primary 11J83. 
of important work by Duffin and Schaffer and in their 1941 paper [2] the condition on $\psi(x)$ is considerably weaker in that merely $\psi(x)$ itself need be non-increasing. The question of the necessary and sufficient condition on $\psi(x)$ was investigated further by Duffin and Schaffer and is the exact subject of the Duffin-Schaffer conjecture. See Chapter 2 of [5] for a treatment of this question.

Glyn Harman has considered the question of restricting the integers $m$ and $n$ to sets of number theoretic interest and these results can be found in Chapter 6 of [5]. In [4] both $m$ and $n$ are restricted to primes and it is in that paper that our present theorem is proved in its one-dimensional form.

We have two things to show in the proof of our theorem; firstly that if the sum (1.2) converges then there are finitely many solutions to the inequalities for almost all $\boldsymbol{\alpha} \in \mathbb{R}^{k}$ and secondly that if the sum diverges then for almost all $\boldsymbol{\alpha} \in \mathbb{R}^{k}$ there are infinitely many solutions. The first implication is proved quite easily using the Borel-Cantelli Lemma and it is the second implication that takes by far the most work.

Definitions. Throughout the paper, $p, q, r$ and $s$, with or without subscripts, will be primes (either positive or negative) unless stated otherwise.

For $\mathbf{x} \in \mathbb{R}^{k}$ let

$$
|\mathbf{x}|=\max _{1 \leq i \leq k}\left(\left|x_{i}\right|\right)
$$

Define $D_{p} \subseteq \mathbb{R}^{k}$ by

$$
D_{p}=\bigcup_{\substack{s_{i} \neq p \\ 1 \leq i \leq k}}\left(\frac{s_{1}-\psi_{1}(p)}{p}, \frac{s_{1}+\psi_{1}(p)}{p}\right) \times \ldots \times\left(\frac{s_{k}-\psi_{k}(p)}{p}, \frac{s_{k}+\psi_{k}(p)}{p}\right),
$$

and

$$
V(N)=\sum_{2 \leq p \leq N} \frac{\psi_{1}(p) \ldots \psi_{k}(p)}{(\log p)^{k}}
$$

Also, let

$$
\mathcal{D}=\bigcap_{q=2}^{\infty} \bigcup_{p=q}^{\infty} D_{p}
$$

that is, the set of points $\boldsymbol{\alpha} \in \mathbb{R}^{k}$ belonging to infinitely many of the $D_{p}$.

Throughout the proof we assume the Prime Number Theorem, the result that the divergence of (1.2) is equivalent to the divergence of

$$
\sum_{p=2}^{\infty} \frac{\psi_{1}(p) \ldots \psi_{k}(p)}{(\log p)^{k}}
$$

and that $\psi_{i}(n) \leq 1 / 2$ for each $i$ and for all $n$. Furthermore we will require the following results: 
RESUlT 1 (First Borel-Cantelli Lemma). If $\left\{A_{n}\right\}$ is a sequence of measurable sets in a measure space whose measures have a finite sum, then the set of points that are in an infinite number of the given sets has measure zero.

RESUlt 2. Let $V$ be an open, Lebesgue-measurable subset of $\mathbb{R}^{k}$. Let $\left(D_{n}\right)$ be a sequence of measurable subsets of $V$. Also, let $\mathcal{D}=\bigcap_{m=1}^{\infty} \bigcup_{n=m}^{\infty} D_{n}$, i.e. the set of points of $V$ belonging to infinitely many of the $D_{n}$. Suppose that for every open cube $C \subseteq V$, we have

$$
\begin{gathered}
\sum_{n=1}^{\infty} \lambda^{k}\left(B_{n}\right)=\infty, \\
\limsup _{N \rightarrow \infty}\left(\sum_{n=1}^{N} \lambda^{k}\left(B_{n}\right)\right)^{2}\left(\sum_{1 \leq m, n \leq N} \lambda^{k}\left(B_{m} \cap B_{n}\right)\right)^{-1} \geq \delta \lambda^{k}(C),
\end{gathered}
$$

where $B_{n}=C \cap D_{n}$ and $\delta>0$ is a constant independent of $C$. Then $\lambda^{k}(V \backslash \mathcal{D})=0$.

For proof of this result see Section 4.

RESUlT 3. Let $\left(\mathbf{x}_{n}\right)$ be a sequence of $N$ points in $\mathbb{R}^{k}$. Let $B$ be a box in $U_{k} \subseteq \mathbb{R}^{k}$ with side lengths $\beta_{1}, \ldots, \beta_{k}$. Then for $\left|\theta_{i}\right| \leq 1(1 \leq i \leq k)$ and positive integer $L$,

$$
\begin{aligned}
& \sum_{\substack{n=1 \\
\mathbf{x}_{n} \in B+\mathbb{Z}^{k}}}^{N} 1 \\
& =\sum_{n=1}^{N}\left(\lambda(B)+1 \cdot \frac{\theta_{1} \mu_{1}(B)}{L+1}+3 \cdot \frac{\theta_{2} \mu_{2}(B)}{(L+1)^{2}}+\ldots+(2 k-1) \frac{\theta_{k} \mu_{k}(B)}{(L+1)^{k}}\right) \\
& \quad+\sum_{0<|\mathbf{m}| \leq L} c(\mathbf{m})\left|\sum_{n=1}^{N} e\left(\mathbf{m} \cdot \mathbf{x}_{n}\right)\right|,
\end{aligned}
$$

where

$$
\mu_{j}(B)=\sum_{\substack{1 \leq i_{a} \leq k \\ i_{b} \neq i_{c}}} \beta_{i_{1}} \ldots \beta_{i_{k-j}},
$$

so e.g. $\mu_{1}(B)=\beta_{1} \beta_{2} \ldots \beta_{k-1}+\ldots+\beta_{2} \beta_{3} \ldots \beta_{k}$, i.e. $\mu_{j}(B)$ is the $(k-j)$ dimensional perimeter of $B$, and with

$$
\begin{aligned}
\mathbf{m} & =\left(m_{1}, \ldots, m_{k}\right), \quad m_{i} \in \mathbb{Z}, \\
c(\mathbf{m}) & =k \prod_{j=1}^{k}\left(\frac{1}{L+1}+\min \left(\frac{1}{\pi\left|m_{j}\right|}, \beta_{j}, 1-\beta_{j}\right)\right) .
\end{aligned}
$$


Proof. This is a $k$-dimensional generalisation of Lemma 5.1 of [4].

For the first implication we assume that the sum

$$
\sum_{p=2}^{\infty} \frac{\psi_{1}(p) \ldots \psi_{k}(p)}{(\log p)^{k}}
$$

converges. Without loss of generality, we will show that the set of $\boldsymbol{\alpha}$ in $\left(\mathbb{R}^{+}\right)^{k}$ for which there are infinitely many solutions to (1.1) has measure zero. Let $C_{\mathbf{M}}=\left[M_{1}, M_{1}+1\right] \times \ldots \times\left[M_{k}, M_{k}+1\right]$ for some vector $\mathbf{M}=\left(M_{1}, \ldots, M_{k}\right)$ $\in \mathbb{Z}^{k}$ and let $B_{p}=C_{M} \cap D_{p}$, where $D_{p}$ is as in the definitions. We have

$$
\begin{aligned}
B_{p}=C_{\mathbf{M}} \cap\left(\bigcup_{\substack{s_{i} \neq p \\
1 \leq i \leq k}}\left(\frac{s_{1}-\psi_{1}(p)}{p}, \frac{s_{1}+\psi_{1}(p)}{p}\right)\right. \\
\left.\quad \times \ldots \times\left(\frac{s_{k}-\psi_{k}(p)}{p}, \frac{s_{k}+\psi_{k}(p)}{p}\right)\right),
\end{aligned}
$$

and we can say that

$$
\sum_{p=2}^{\infty} \lambda^{k}\left(B_{p}\right) \leq \sum_{p=2}^{\infty} \frac{2^{k} \psi_{1}(p) \ldots \psi_{k}(p)}{p^{k}} \sum_{s_{1} \sim p} \ldots \sum_{s_{k} \sim p} 1,
$$

where $s_{i} \sim p$ denotes $p M_{i}<s_{i}<p\left(M_{i}+1\right)$. Hence using the BrunTitchmarsh inequality for the number of primes in an interval of length $p$ we can say that the sum in question is

$$
\leq C(k) \sum_{p=2}^{\infty} \frac{\psi_{1}(p) \ldots \psi_{k}(p)}{(\log p)^{k}} .
$$

Since the sum above converges the sum of the measures of the $B_{p}$ is finite and so by the First Borel-Cantelli Lemma (Result 1) we can conclude that the set of $\boldsymbol{\alpha}$ contained in infinitely many $B_{p}$ has measure zero. Now the set of $\boldsymbol{\alpha}$ contained in infinitely many $D_{p}$ is just the union over all $\mathbf{M}$ of those contained in infinitely many $C_{\mathbf{M}} \cap D_{p}$ and since a countable union of null sets is itself null we can conclude that this too is a null set. From this the result follows.

2. Construction. We now assume that

$$
\sum_{p=2}^{\infty} \frac{\psi_{1}(p) \ldots \psi_{k}(p)}{(\log p)^{k}}
$$

diverges. We will show that the sequence $\left(D_{p}\right)$ of measurable sets satisfy the conditions of Result 2 with $V=\left(\mathbb{R}^{+}\right)^{k}$. We will then be able to conclude that the set of $\boldsymbol{\alpha}$ contained in infinitely many of the $D_{p}$ has full measure, which is equivalent to the assertion that for almost all $\boldsymbol{\alpha}$ there are infinitely 
many solutions to the simultaneous inequalities (1.1). This then proves the theorem. It follows from the Prime Number Theorem that if (1.2) diverges then

$$
\sum_{p=2}^{\infty} \lambda^{k}\left(D_{p} \cap C\right)=\infty
$$

where $C$ is any cube in $\left(\mathbb{R}^{+}\right)^{k}$. Thus (1.3) holds, and this is the first condition of Result 2.

We now need to show that the second condition of Result 2 holds. Thus we show that given any $C \subseteq\left(\mathbb{R}^{+}\right)^{k}$, we have

$$
\limsup _{N \rightarrow \infty}\left(\sum_{p=2}^{N} \lambda^{k}\left(D_{p} \cap C\right)\right)^{2}\left(\sum_{2 \leq p, q \leq N} \lambda^{k}\left(D_{p} \cap D_{q} \cap C\right)\right)^{-1}
$$

$\geq \delta \lambda^{k}(C)$,

where $\delta>0$ is a constant independent of the choice of $C$. To show this we prove the following lemma.

Lemma 1. For $C \subseteq\left(\mathbb{R}^{+}\right)^{k}$ we have

$$
\lim _{N \rightarrow \infty} \frac{1}{V(N)} \sum_{p=2}^{N} \lambda^{k}\left(D_{p} \cap C\right)=2^{k} \lambda^{k}(C)
$$

and

$$
\limsup _{N \rightarrow \infty} \frac{1}{(V(N))^{2}} \sum_{2 \leq p, q \leq N} \lambda^{k}\left(D_{p} \cap D_{q} \cap C\right) \leq \Delta \lambda^{k}(C),
$$

where $\Delta>0$ is independent of the choice of $C$.

Proof. For ease of notation we prove the lemma for cubes $C$ of the form $(1,1+\theta)^{k}$ with $\theta \leq 1 / 2$ rather than for cubes with a corner at some general point $\boldsymbol{\eta}$ of $\left(\mathbb{R}^{+}\right)^{k}$. Also, note that $\Delta$ will be used to denote a positive real, independent of the cube $C$, and will not necessarily be the same at every occurrence. To show (2.2) note that

$$
\sum_{p=2}^{N} \lambda^{k}\left(D_{p} \cap C\right)=\sum_{p=2}^{N} 2^{k} \frac{\psi_{1}(p) \ldots \psi_{k}(p)}{p^{k}} \sum_{s_{1} \sim p} \ldots \sum_{s_{k} \sim p} 1
$$

where $s_{i} \sim p$ denotes $p<s_{i}<p(1+\theta)$. By the Prime Number Theorem, this is

$$
\sum_{p=2}^{N} 2^{k} \frac{\psi_{1}(p) \ldots \psi_{k}(p)}{p^{k}} \cdot \frac{\theta^{k} p^{k}}{(\log p)^{k}}(1+o(1))
$$

and this is just $2^{k} \theta^{k} V(N)+o(V(N))$ as required. 
For (2.3) note that since

$$
\sum_{2 \leq p=q \leq N} \lambda^{k}\left(D_{p} \cap D_{q} \cap C\right)=2^{k} \theta^{k} V(N)+o(V(N))
$$

and the right-hand side above is $o(V(N))^{2}$, we need only show that

$$
\sum_{2 \leq p<q \leq N} \lambda^{k}\left(D_{p} \cap D_{q} \cap C\right) \leq \Delta \lambda^{k}(C)(V(N))^{2}+o(V(N))^{2} .
$$

We will denote the sum on the left-hand side above by $S(N)$. Now $D_{q}$ is a union of cubes each having a measure of

$$
2^{k} \frac{\psi_{1}(q) \ldots \psi_{k}(q)}{q^{k}}
$$

Since this is also the maximum measure of the intersection of a cube in $D_{p}$ and a cube in $D_{q}$, where $p<q$, we may write an upper bound on $\lambda^{k}\left(D_{p} \cap D_{q} \cap C\right)$ by bounding the number of such intersections and counting them with this weight. Suppose that

$$
\left(\frac{s_{1}-\psi_{1}(p)}{p}, \frac{s_{1}+\psi_{1}(p)}{p}\right) \times \ldots \times\left(\frac{s_{k}-\psi_{k}(p)}{p}, \frac{s_{k}+\psi_{k}(p)}{p}\right)
$$

intersects with

$$
\left(\frac{s_{1}-\psi_{1}(q)}{q}, \frac{s_{1}+\psi_{1}(q)}{q}\right) \times \ldots \times\left(\frac{s_{k}-\psi_{k}(q)}{q}, \frac{s_{k}+\psi_{k}(q)}{q}\right):
$$

A necessary condition for this is given by

$$
0<\left|r_{i} p-s_{i} q\right|<2 q \psi_{i}(p) \quad \text { for } i=1, \ldots, k ;
$$

i.e. that the centres of the two cubes are sufficiently close for intersection to occur. (Note that we need not consider equality with zero in the above expression since we are assuming here that $p \neq q$ and in our definition of $D_{p}, s_{i} \neq p$.) Since we are only counting intersections which lie in $C$ the centres of both cubes must certainly lie in $C$ and so we have the condition $\mathbf{r} \sim q$, meaning that $q<r_{i}<q(1+\theta)$ for each $i$ between 1 and $k$, and $\mathbf{r} \sim p$ similarly. This argument gives the following sum as an upper bound:

$$
S(N) \leq \sum_{2 \leq p<q \leq N} 2^{k} \frac{\psi_{1}(q) \ldots \psi_{k}(q)}{q^{k}} \sum_{\mathbf{r} \sim q} \sum_{\mathbf{s} \sim p}^{*} 1
$$

the asterix denoting the summation condition given in (2.5). We now split up the range $2 \leq p<q \leq N$ into subranges by defining parameters $P$ and $Q$ as taking values $2(1+\theta)^{n}$ in the range $(2, N(1+\theta))$. Using the " $~$ " notation introduced above we can rewrite the sum as

$$
S(N) \leq \sum_{2 \leq P \leq Q \leq N} 2^{k} \frac{\psi_{1}(Q) \ldots \psi_{k}(Q)}{Q^{k}} \sum_{\substack{\mathbf{r}: Q \\ q \sim Q}} \sum_{\substack{\mathbf{s}: P \\ p \sim P}}^{*} 1
$$


Here, $\mathbf{r}: Q$ denotes $Q<r_{i}<Q(1+\theta)^{2}$ for $i=1, \ldots, k$ and the asterix denotes the following conditions:

$$
0<\left|r_{i} p-s_{i} q\right|<3 Q \psi_{i}(P) \quad \text { for } i=1, \ldots, k .
$$

Now define $S(P, Q)$ to be the double sum part of $(2.6) . S(P, Q)$ counts the number of solutions to a set of $k$ inequalities involving integers alone and the proof of (2.3) and hence of the main theorem now rests on proving a suitable size upper bound for this.

\section{Main lemma}

Lemma 2. For $Q \geq P \geq \theta^{-2}$ there exists a small, positive real $c$ (dependent only on the $\psi$ functions), such that

$$
S(P, Q) \leq \frac{\Delta \theta^{k+2} P Q H_{1} \ldots H_{k}}{(\log Q)^{k+1}(\log P)^{k+1}}+O\left(H_{1} \ldots H_{k} P Q^{1-c}\right) .
$$

Here $\Delta$ is a positive constant dependent only on the dimension $k$ and $H_{i}=$ $3 Q \psi_{i}(P)$.

The criterion of Lemma 2 is that $Q \geq P \geq \theta^{-2}$; when this does not hold either (1) $Q<\theta^{-2}$ or (2) $P \leq \theta^{-2}<Q$. In either case we have, trivially,

$$
S(P, Q) \leq \sum_{p \sim P} \sum_{q \sim Q} \sum_{\mathbf{r}: Q} \sum_{\mathbf{s}: P} 1
$$

In case (1) this is just $O(1)$ with respect to $N$ while in case (2) it is

$$
\ll \frac{Q^{k+1}}{(\log P)^{k+1}(\log Q)^{k+1}} .
$$

Hence by (2.6), the contribution this gives to $S(N)$ has size

$$
\ll \sum_{\substack{2 \leq P \leq Q \leq N \\ P \leq \theta^{-2}}} \frac{Q \psi_{1}(Q) \ldots \psi_{k}(Q)}{(\log P)^{k+1}(\log Q)^{k+1}}
$$

which is $O(V(N))$. By this and Lemma 2 then, $S(N)$ is

$$
\leq \Delta \theta^{k} \sum_{2 \leq P \leq Q \leq N} \frac{P Q \theta^{2} \psi_{1}(P) \ldots \psi_{k}(P) \psi_{1}(Q) \ldots \psi_{k}(Q)}{(\log P)^{k+1}(\log Q)^{k+1}}+o(V(N))^{2}
$$

and this is

$$
\leq \Delta \lambda^{k}(C)(V(N))^{2}+o(V(N))^{2}
$$

Hence (2.4) holds, which directly implies (2.3), thus proving the theorem. 
In Lemma 2 we are looking for an upper bound on the number of solutions in primes to the inequalities

$$
\begin{aligned}
& 0<\left|r_{1} p-s_{1} q\right|<H_{1}, \\
& \ldots \ldots \ldots \ldots \ldots \ldots \ldots . \cdots \\
& 0<\left|r_{k} p-s_{k} q\right|<H_{k},
\end{aligned}
$$

where $r_{i}: Q, s_{i}: P, p \sim P$ and $q \sim Q$.

We begin by proving the lemma in the case $k=2$. This serves to illustrate all the new difficulties introduced when generalising the one-dimensional result. We shall then give some details for the case $k>2$. The proof falls into three parts. The first is when the $P$ variable is smaller than a power of $Q$ (we will take $P<Q^{1 / 4}$ ) and in this case the Brun-Titchmarsh inequality provides a suitable bound. The other two parts cover the case $P \geq Q^{1 / 4}$ and are distinguished by the following assumption on the relative sizes of our $\psi(P)$ functions:

$$
\exists \delta>0 \text { such that for } i=1 \text { to } k, \quad \psi_{i}(P) \gg P^{\delta-1} .
$$

The second part will assume that the above holds, while the third part, when it fails will be similar to the $k=1$ version of the lemma which is proved in [2] using a result on incomplete Kloosterman sums. The latter two parts use the following sieve results.

Lemma 3a. For squarefree integer $d$ and positive integer $\kappa$, write

$$
\omega(d)=\prod_{p \mid d} \kappa .
$$

Let $\mathcal{A}$ be a set of positive integers and $Y, X$ positive reals. Put

$$
R_{d}=\sum_{\substack{n \in \mathcal{A} \\ d \mid n}} 1-\frac{\omega(d)}{d} X .
$$

Then the number of members of $\mathcal{A}$ having all their prime factors greater than $Y$ is

$$
\leq \frac{\Delta X}{(\log Y)^{\kappa}}+O\left(\sum_{d \leq Y} \mu^{2}(d) d^{\varepsilon}\left|R_{d}\right|\right) .
$$

Here $\mu(d)$ is the Möbius function, $\varepsilon$ is an arbitrary positive real and $\Delta i s$ a positive constant.

Proof. This is a special case of Theorem (5.2) of [3].

Lemma 3b. For squarefree integer $d$ and positive integer $\kappa$, write

$$
\omega(d)=\prod_{p \mid d}\left(\kappa-\frac{1}{p}\right)=\sum_{\substack{x_{1} \ldots x_{\kappa}=d \\ x_{1}, \ldots, x_{\kappa} \geq 0}} \frac{\phi\left(x_{1}\right)}{x_{1}} .
$$


Let $\mathcal{A}$ be a set of positive integers, $Y, X$ positive reals and $h$ an even positive integer. Put

$$
R_{d}=\sum_{\substack{n \in \mathcal{A} \\ d \mid n}} 1-\frac{\omega(d)}{d} X
$$

Then the number of members of $\mathcal{A}$ having all their prime factors either dividing $h$ or greater than $Y$ is

$$
\leq \frac{\Delta X}{(\log Y)^{\kappa}}\left(\frac{h}{\phi(h)}\right)^{\kappa}+O\left(\sum_{\substack{d \leq Y \\(d, h)=1}} \mu^{2}(d) d^{\varepsilon}\left|R_{d}\right|\right) .
$$

Here $\mu(d)$ is the Möbius function, $\phi(h)$ is the Euler totient function, $\varepsilon$ is an arbitrary positive real and $\Delta$ is a positive constant.

Proof. This is a special case of Theorem (5.2) of [3].

These sieve results will in fact give an upper bound on the number of solutions to the inequalities in integers $s_{i}, r_{i}$ with prime factors larger than a given size so in this section $s_{i}, r_{i}$ are not necessarily primes.

We require one more auxiliary lemma before beginning the proof of Lemma 2.

LEMMA 4. Let $U, V \geq 1$ be real and $t, w$ integers with $(t, w)=1$ and $V \leq 3 w$. Then for any $\varepsilon>0$, we have

$$
\sum_{1 \leq u \leq U}\left|\sum_{\substack{1 \leq v \leq V \\(v, w)=1}} e\left(\frac{\bar{v} u t}{w}\right)\right| \ll U w^{1 / 2+\varepsilon} .
$$

Here $\bar{v}$ denotes a solution of $x v \equiv 1(\bmod w)$.

Proof. See Lemmas 6 and 7 in Chapter 2 of [6].

Proof of Lemma 2. CASE 1: $P<Q^{1 / 4}$. Fix $s_{1}, p$ and $q$ in the first equation, then re-arranging the equation shows that the number of solutions is

$$
\pi\left(\frac{s_{1} q}{p}+\frac{H_{1}}{p}\right)-\pi\left(\frac{s_{1} q}{p}-\frac{H_{1}}{p}\right) .
$$

By the Brun-Titchmarsh inequality this is

$$
\leq \frac{4 H_{1}}{p \log \left(H_{1} / p\right)} \text {. }
$$

Now since $P<Q^{1 / 4}$ and $H_{1} \gg Q / P$ this is

$$
\ll \frac{4 H_{1}}{P \log Q} \text {. }
$$

Since we get a similar bound by fixing $s_{2}$ in the second equation, the total number of solutions is 


$$
\ll \sum_{p \sim P} \sum_{q \sim Q} \sum_{s_{1}: P} \sum_{s_{2}: P} \frac{H_{1}}{P \log Q} \cdot \frac{H_{2}}{P \log Q},
$$

which is

$$
\leq \frac{\Delta \theta^{4} P Q H_{1} H_{2}}{(\log Q)^{3}(\log P)^{3}}
$$

using the fact that $P \theta \geq P^{1 / 2}$ and $Q \theta \geq Q^{1 / 2}$. This proves Case 1 of the lemma.

CASE 2: $P \geq Q^{1 / 4}$ and there exists $\delta>0$ such that $\psi_{i}(P) \gg P^{\delta-1}$, $i=1,2$. We fix $q$ and apply Lemma 3 a with $\kappa=4$ and with

$$
\mathcal{A}=\left\{r_{1} r_{2} s_{1} s_{2}|| r_{i} p-s_{i} q \mid<H_{i}, r_{i}: Q, s_{i}: P \text { and } p \sim P\right\} .
$$

Note that $p$ is prime here. In order to estimate the size of $R_{d}$ we need to look at the size of

$$
\mathcal{A}_{d}=\{n \in \mathcal{A}|d| n\} .
$$

For this we will obtain an asymptotic formula using the Fourier analysis Result 3. Writing a member of $\mathcal{A}_{d}$ in the form

$$
\left(r_{1} x_{1}\right)\left(r_{2} x_{2}\right)\left(s_{1} y_{1}\right)\left(s_{2} y_{2}\right)
$$

where $x_{1} x_{2} y_{1} y_{2}=d$, we can obtain the number of elements in $\mathcal{A}_{d}$ by counting solutions to

$$
\left\|\frac{r_{1} x_{1} p}{q y_{1}}\right\|<\frac{H_{1}}{q y_{1}}, \quad\left\|\frac{r_{2} x_{2} p}{q y_{2}}\right\|<\frac{H_{2}}{q y_{2}},
$$

where $\|\cdot\|$ denotes distance to the nearest integer. Using Result 3 with

$$
B=\left(-\frac{H_{1}}{q y_{1}}, \frac{H_{1}}{q y_{1}}\right) \times\left(-\frac{H_{2}}{q y_{2}}, \frac{H_{2}}{q y_{2}}\right)
$$

we get

$$
\begin{aligned}
\sum_{n \in \mathcal{A}_{d}} 1=\sum_{x_{1} x_{2} y_{1} y_{2}=d}\left(\sum _ { r _ { 1 } , r _ { 2 } , p } \left(\frac{4 H_{1} H_{2}}{y_{1} y_{2} q^{2}}+\frac{\theta_{1}}{L+1}\left(\frac{2 H_{1}}{y_{1} q}+\frac{2 H_{2}}{y_{2} q}\right)\right.\right. \\
\left.\left.+\frac{3 \theta_{2}}{(L+1)^{2}}\right)+\theta_{3} E_{3}\right) .
\end{aligned}
$$

In the above sum, $r_{1}, r_{2}$ and $p$ lie in the following ranges:

$$
r_{1}:\left(\frac{Q}{x_{1}}\right), \quad r_{2}:\left(\frac{Q}{x_{2}}\right), \quad p \sim P .
$$

We let $E_{3}$ denote the exponential sum part of Result 3 and we will deal with this presently. Of the three summands within the $r_{1}, r_{2}, p$ sum it is the first which will form the main term and the other terms we will label $E_{1}$ and $E_{2}$ respectively. The main term then is 


$$
\sum_{x_{1} x_{2} y_{1} y_{2}=d} \frac{4 H_{1} H_{2}}{y_{1} y_{2} q^{2}}\left(\frac{\theta(2+\theta) Q}{x_{1}}+O(1)\right)\left(\frac{\theta(2+\theta) Q}{x_{2}}+O(1)\right) \pi(P, \theta)
$$

where $\pi(P, \theta)$ denotes the number of primes in the interval $(P, P(1+\theta))$. The above is

$$
\begin{aligned}
\frac{4 H_{1} H_{2} \theta^{2}(2+\theta)^{2} Q^{2} \pi(P, \theta)}{q^{2}} \sum_{x_{1} x_{2} y_{1} y_{2}=d} & \frac{1}{x_{1} x_{2} y_{1} y_{2}} \\
+ & O\left(\frac{H_{1} H_{2} P Q}{q^{2}} \sum_{x_{1} x_{2} y_{1} y_{2}=d} \frac{1}{x_{1} y_{1} y_{2}}\right)
\end{aligned}
$$

and can be written as

$$
\frac{\omega(d)}{d} X+O\left(\frac{H_{1} H_{2} P d^{\varepsilon}}{q}\right) .
$$

Hence we can say that

$$
\left|R_{d}\right|=O\left(\frac{H_{1} H_{2} P d^{\varepsilon}}{q}\right)+E_{1}+E_{2}+E_{3} .
$$

Now letting $L=Q$, we have

$$
E_{1}=\sum_{x_{1} x_{2} y_{1} y_{2}=d} \sum_{r_{1}, r_{2}, p} \frac{\theta_{1}}{L+1}\left(\frac{2 H_{1}}{y_{1} q}+\frac{2 H_{2}}{y_{2} q}\right) .
$$

This has order no larger than $\left(H_{1}+H_{2}\right) P d^{\varepsilon}$, which in turn is

$$
\ll \frac{H_{1} H_{2} P d^{\varepsilon}}{Q^{\delta}},
$$

since we have assumed that there exists $\delta>0$ such that $\psi_{i}(P) \gg P^{\delta-1}$.

The next error term, $E_{2}$, is

$$
\sum_{x_{1} x_{2} y_{1} y_{2}=d} \sum_{r_{1}, r_{2}, p} \frac{3 \theta_{2}}{(L+1)^{2}} .
$$

This can easily be seen to have order no worse than

$$
\frac{H_{1} H_{2} P d^{\varepsilon}}{Q} \text {. }
$$

The exponential sum term, $E_{3}$, is

$$
\sum_{x_{1} x_{2} y_{1} y_{2}=d} \sum_{0<|\mathbf{m}| \leq L} c(\mathbf{m})\left|\sum_{r_{1}, r_{2}, p} e\left(\frac{m_{1} r_{1} x_{1} p}{y_{1} q}+\frac{m_{2} r_{2} x_{2} p}{y_{2} q}\right)\right| .
$$

Here $c(\mathbf{m})$ (see Result 3) is

$$
2 \prod_{j=1}^{2}\left(\frac{1}{L+1}+\min \left(\frac{1}{\pi\left|m_{j}\right|}, \beta_{j}, 1-\beta_{j}\right)\right) .
$$


We are taking

$$
\beta_{j}=\frac{2 H_{j}}{q y_{j}}
$$

and the order of $c(\mathbf{m})$ will depend on the size of $\left|m_{j}\right|$ relative to this. Note that we have already set $L=Q$. Summing over $r_{1}$ and $r_{2}$ we can say that $E_{3}$ is

$$
\leq \sum_{x_{1} x_{2} y_{1} y_{2}=d} \sum_{0<|\mathbf{m}| \leq Q} \sum_{p \sim P} c(\mathbf{m}) \min \left(\frac{Q}{x_{1}}, \frac{1}{\left\|\frac{m_{1} x_{1} p}{q y_{1}}\right\|}\right) \min \left(\frac{Q}{x_{2}}, \frac{1}{\left\|\frac{m_{2} x_{2} p}{q y_{2}}\right\|}\right) .
$$

The largest term in the above expression arises when $m_{1}$ or $m_{2}$ are zero. Without loss of generality, let $m_{2}=0$; then we have a term which is

$$
\ll \sum_{x_{1} x_{2} y_{1} y_{2}=d} \sum_{1 \leq\left|m_{1}\right| \leq Q} \sum_{p \sim P} \min \left(\frac{H_{1}}{q}, \frac{1}{\left|m_{1}\right|}\right) \frac{H_{2}}{q y_{2}} \cdot \frac{Q}{x_{2}} \cdot \frac{1}{\left\|\frac{m_{1} x_{1} p}{q y_{1}}\right\|} .
$$

Splitting the range of $m_{1}$ into two sections shows that this is

$$
\begin{aligned}
& \ll H_{2} \sum_{x_{1} x_{2} y_{1} y_{2}=d} \frac{1}{x_{2} y_{2}} \sum_{\substack{1 \leq\left|m_{1}\right| \leq Q / H_{1} \\
p \sim P}} \frac{H_{1}}{Q} \cdot \frac{1}{\left\|\frac{m_{1} x_{1} p}{q y_{1}}\right\|} \\
& +H_{2} \sum_{x_{1} x_{2} y_{1} y_{2}=d} \frac{1}{x_{2} y_{2}} \sum_{\substack{Q / H_{1} \leq\left|m_{1}\right| \leq Q \\
p \sim P}} \frac{1}{\left|m_{1}\right|} \cdot \frac{1}{\left\|\frac{m_{1} x_{1} p}{q y_{1}}\right\|} .
\end{aligned}
$$

Combining $m_{1}$ with $p$ to give a single variable $a$ we can say that this is

$$
\begin{aligned}
& \ll \frac{H_{1} H_{2}}{Q} \sum_{x_{1} x_{2} y_{1} y_{2}=d} \frac{1}{x_{2} y_{2}} \sum_{P \leq a<2 P Q / H_{1}} \frac{1}{\left\|\frac{a x_{1} \|}{q y_{1}}\right\|} \\
& +\frac{H_{1} H_{2}}{Q} \sum_{x_{1} x_{2} y_{1} y_{2}=d} \frac{1}{x_{2} y_{2}} \sum_{k=0}^{2 \log Q} \frac{1}{2^{k}} \sum_{2^{k} P Q / H_{1} \leq a<2^{k+1} P Q / H_{1}} \frac{1}{\left\|\frac{a x_{1}}{q y_{1}}\right\|} .
\end{aligned}
$$

This in turn is

$$
\begin{aligned}
& \ll \frac{H_{1} H_{2}}{Q} \sum_{x_{1} x_{2} y_{1} y_{2}=d} \frac{1}{x_{2} y_{2}}\left(\frac{2 P}{H_{1}}+1\right) Q \log Q \\
& \quad+\frac{H_{1} H_{2}}{Q} \sum_{x_{1} x_{2} y_{1} y_{2}=d} \frac{1}{x_{2} y_{2}} \sum_{k=0}^{2 \log Q} \frac{1}{2^{k}}\left(\frac{2^{k} P}{H_{1}}+1\right) Q \log Q
\end{aligned}
$$

which is

$$
\ll H_{2} P d^{\varepsilon} \log ^{2} Q+H_{1} H_{2} d^{\varepsilon} \log Q .
$$

Now since $\psi_{1}(P) \gg P^{\delta-1}$, this is

$$
\ll \frac{H_{1} H_{2} P d^{\varepsilon}}{Q^{\beta}}\left(\log ^{2} Q\right),
$$


where $\beta=\min (\delta, 1 / 4)$. The remaining part of $E_{3}$ has $m_{1}$ and $m_{2}$ both non-zero and for this part we start by noting that for any fixed $p$,

$$
\sum_{1 \leq\left|m_{2}\right| \leq Q} \frac{1}{\left\|\frac{m_{2} x_{2} p}{y_{2} q}\right\|} \ll Q y_{2} \log Q .
$$

Using this bound for the $m_{2}$ part of the double exponential sum (having $m_{1}$ and $m_{2}$ both non-zero) shows that the sum is no more than

$$
Q \log Q \sum_{x_{1} x_{2} y_{1} y_{2}=d} y_{2} \sum_{1 \leq m_{1} \leq Q} \frac{H_{2}}{q} \min \left(\frac{H_{1}}{q}, \frac{1}{\left|m_{1}\right|}\right) \sum_{p \sim P} \frac{1}{\left\|\frac{m_{1} x_{1} p}{y_{1} q}\right\|} .
$$

As before we can now combine the two variables $m_{1}$ and $p$ to give a single variable $a$ to get our bound. This is straightforward for $\left|m_{1}\right| \leq q / H_{1}$ and uses a splitting up argument for $m_{1}$ in the range $q / H_{1}<\left|m_{1}\right|<Q$. Together these give a bound no more than

$$
\frac{H_{1} H_{2} P d^{1+\varepsilon}}{Q^{\beta}}\left(\log ^{3} Q\right) .
$$

Looking at (3.2) and the bounds we have obtained for $E_{1}, E_{2}$ and $E_{3}$ in (3.3)-(3.6) respectively, we can say that

$$
\left|R_{d}\right| \ll \frac{H_{1} H_{2} P d^{1+\varepsilon}}{Q^{\beta}}\left(\log ^{2} Q\right) .
$$

This means that the error term of the sieve is

$$
\sum_{d \leq Y} \mu^{2}(d) d^{\varepsilon}\left|R_{d}\right| \leq \frac{\Delta H_{1} H_{2} P Y^{2+\varepsilon}}{Q^{\beta-\eta}},
$$

for any small $\eta>0$, thus accounting for the $\log ^{2} Q$ term in (3.7). Let $Y=Q^{\alpha}$. Then taking $\alpha=\eta=\varepsilon=\beta / 4$ makes this term

$$
\leq \Delta \frac{H_{1} H_{2} P}{Q^{c}},
$$

where $c=\beta / 8$ say. By Lemma 3 the size of $\mathcal{A}$ is

$$
\leq \frac{\Delta X}{(\log Q)^{4}}+O\left(\frac{H_{1} H_{2} P}{Q^{c}}\right)
$$

So summing over $q$ we can say that the $S(P, Q)$ of Lemma 2 is

$$
\leq \frac{\Delta \pi(P, \theta) H_{1} H_{2} \theta^{2}(2+\theta)^{2} Q^{2}}{(\log Q)^{4}} \sum_{q \sim Q} \frac{1}{q^{2}}+O\left(H_{1} H_{2} P Q^{1-c}\right),
$$

which is

$$
\leq \frac{\Delta P \theta^{3}}{(\log P)} \cdot \frac{H_{1} H_{2}}{(\log Q)^{4}} \cdot \frac{\theta Q}{(\log Q)}+O\left(H_{1} H_{2} P Q^{1-c}\right) .
$$

This completes Case 2 of the proof of Lemma 2. 
CASE 3: $P \geq Q^{1 / 4}$ and there exists $i$ such that $\psi_{i}(P) \ll P^{\delta-1}$ for all $\delta>0$. Without loss of generality assume $\psi_{1}(P) \ll P^{\delta-1}$ for all $\delta>0$ (and consequently that there exists $\delta^{\prime}>0$ such that $\psi_{2}(P) \gg P^{\delta^{\prime}-1}$ ). The number of solutions to the equations for those $h_{1}=\left|r_{1} p-s_{1} q\right|$ with $p \mid h_{1}$ is $\ll H_{1} H_{2} P \ll H_{1} H_{2} P Q^{3 / 4}$. For other values of $h_{1}$ we fix $h_{1}$ and $p$ and apply the sieve result, Lemma $3 \mathrm{~b}$, with $\kappa=5$ and with

$$
\mathcal{A}=\left\{r_{1} r_{2} s_{1} s_{2} q\left|r_{1} p-s_{1} q=h_{1},\right| r_{2} p-s_{2} q \mid<H_{2}, s_{i}: P \text { and } q \sim Q\right\} .
$$

Note that here we are including $q$ in the sieve so we are not restricting $q$ to being prime. We want to obtain a suitable bound on the size of

$$
\mathcal{A}_{d}=\{n \in \mathcal{A}|d| n\} .
$$

For squarefree $d$ this is equivalent to the number of solutions to

$$
\begin{array}{cl}
x_{1} x_{2} y_{1} y_{2} z=d, \quad x_{1}, \ldots, z \geq 1, \\
r_{1} x_{1} p-s_{1} y_{1} q z=h_{1} \quad \text { and } \quad\left|r_{2} x_{2} p-s_{2} y_{2} q z\right|<H_{2} .
\end{array}
$$

Since we are considering $d$ with $\left(h_{1}, d\right)=1$ in this sieve, we also have $\left(h_{1}, x_{1} p\right)=1$ and consequently $\left(s_{1} y_{1} z, x_{1} p\right)=1$. Thus the above is equivalent to

$$
\begin{aligned}
& x_{1} x_{2} y_{1} y_{2} z=d, \quad x_{1}, \ldots, z \geq 1, \\
& \overline{s_{1} y_{1} z} h_{1}+q \equiv 0\left(\bmod x_{1} p\right) \text { and } s_{2} y_{2} q z+h_{2} \equiv 0\left(\bmod x_{2} p\right) \text {. }
\end{aligned}
$$

Here the $\bar{a}$ notation denotes the inverse of $a$ modulo $x_{1} p$. We now convert the congruences to exponential sums and say that the number of solutions to the above is equal to

$$
\sum_{x_{1} x_{2} y_{1} y_{2} z=d} \frac{1}{p^{2} x_{1} x_{2}} \sum_{\substack{0 \leq\left|m_{1}\right| \leq p x_{1} / 2 \\ 0 \leq\left|m_{2}\right| \leq p x_{2} / 2}} \sum_{q \sim Q / z} \sum_{\substack{s_{1}: P / y_{1} \\\left(s_{1}, p x_{1}\right)=1}} \sum_{\substack{s_{2}: P / y_{2} \\ 0<\left|h_{2}\right| \leq H_{2}}} S
$$

(with the convention that the value $m_{i}=-p x_{i} / 2$ is omitted from the summation if $p x_{i}$ is even), and where

$$
S=e\left(\frac{m_{1}\left(\overline{s_{1} y_{1} z} h_{1}+q\right)}{x_{1} p}\right) e\left(\frac{m_{2}\left(s_{2} y_{2} z q+h_{2}\right)}{x_{2} p}\right) .
$$

The part of this sum with $m_{1}=m_{2}=0$ is our main term and is equal to

$$
X \frac{\omega(d)}{d}+O\left(\frac{H_{2} Q d^{\varepsilon}}{p}\right)
$$

where $X=2 H_{2} P^{2} Q \theta^{3}(2+\theta)^{2} / p^{2}$. The remainder term in the sieve, $R_{d}$, is thus 


$$
\begin{aligned}
\sum_{x_{1} x_{2} y_{1} y_{2} z=d} \frac{1}{p^{2} x_{1} x_{2}} \sum_{\begin{array}{c}
0 \leq\left|m_{1}\right| \leq p x_{1} / 2 \\
0 \leq\left|m_{2}\right| \leq p x_{2} / 2 \\
m_{1}, m_{2} \text { not both } 0
\end{array}} \sum_{q \sim Q / z} \sum_{\substack{s_{1}: P / y_{1} \\
\left(s_{1}, p x_{1}\right)=1}} \sum_{\begin{array}{c}
s_{2}: P / y_{2} \\
0<\left|h_{2}\right| \leq H_{2}
\end{array}} S \\
+O\left(H_{2} Q d^{\varepsilon}\right) .
\end{aligned}
$$

We now consider the parts of this sum where either $m_{1}$ or $m_{2}$ are zero. Firstly, the part with $m_{1}=0$ is

$$
\begin{aligned}
& \ll \frac{1}{p} \sum_{x_{1} x_{2} y_{1} y_{2} z=d} \frac{1}{x_{1} x_{2} y_{1}} \\
& \times \sum_{1 \leq\left|m_{2}\right| \leq p x_{2} / 2} \mid \sum_{\substack{q \sim Q / z \\
s_{2}: P / y_{2}}} \sum_{0<\left|h_{2}\right| \leq H_{2}} e\left(\frac{m_{2}\left(s_{2} y_{2} z q+h_{2}\right)}{x_{2} p}\right) .
\end{aligned}
$$

Now since

$$
\left|\sum_{0<\left|h_{2}\right| \leq H_{2}} e\left(\frac{m_{2} h_{2}}{x_{2} p}\right)\right| \leq \min \left(H_{2}, \frac{x_{2} p}{\left|m_{2}\right|}\right),
$$

the exponential sum above is

$$
\begin{aligned}
& \ll \frac{1}{p} \sum_{x_{1} x_{2} y_{1} y_{2} z=d} \frac{1}{x_{1} x_{2} y_{1}} \\
& \quad \times \sum_{1 \leq\left|m_{2}\right| \leq p x_{2} / 2} \min \left(H_{2}, \frac{x_{2} p}{\left|m_{2}\right|}\right) \sum_{q \sim Q / z}\left|\sum_{s_{2}: P / y_{2}} e\left(\frac{m_{2} s_{2} y_{2} z q}{x_{2} p}\right)\right| .
\end{aligned}
$$

We now sum over $s_{2}$ and split the sum into two parts distinguished by the size of $m_{2}$ to give the following bound:

$$
\begin{aligned}
\frac{H_{2}}{p} \sum_{x_{1} x_{2} y_{1} y_{2} z=d} \frac{1}{x_{1} x_{2} y_{1}} \sum_{1 \leq\left|m_{2}\right| \leq p x_{2} / H_{2}} \sum_{q \sim Q / z} \frac{1}{\left\|\frac{m_{2} y_{2} z q}{x_{2} p}\right\|} \\
+\sum_{x_{1} x_{2} y_{1} y_{2} z=d} \frac{1}{x_{1} y_{1}} \sum_{p x_{2} / H_{2} \leq\left|m_{2}\right| \leq p x_{2} / 2} \frac{1}{\left|m_{2}\right|} \sum_{q \sim Q / z} \frac{1}{\left\|\frac{m_{2} y_{2} z q}{x_{2} p}\right\|} .
\end{aligned}
$$

We then combine $m_{2}$ with $q$ giving a single variable $a$ running over a large range. This will produce a $\sum_{d \mid a} 1$ term which adds a factor of $Q^{\varepsilon}$ to the bound. The bound we obtain by doing this is

$$
\begin{aligned}
& \sum_{x_{1} x_{2} y_{1} y_{2} z=d} \frac{1}{x_{1} y_{1} z} Q^{1+\varepsilon} \log P \\
& \quad+\left(\sum_{x_{1} x_{2} y_{1} y_{2} z=d} \frac{1}{x_{1} y_{1} z} Q^{1+\varepsilon} \log P \log H_{2}+\sum_{x_{1} x_{2} y_{1} y_{2} z=d} \frac{1}{x_{1} y_{1}} H_{2} Q^{\varepsilon} \log P\right) .
\end{aligned}
$$


This is no more than $Q Q^{\varepsilon} d^{\varepsilon}$, and since $H_{2} \gg Q P^{-\delta^{\prime}}$, the bound is

$$
\ll H_{2} P^{\delta^{\prime}} Q^{\varepsilon} d^{\varepsilon} \text {. }
$$

Next we need to bound the part of (3.9) that has $m_{2}=0$. This is

$$
\begin{aligned}
& \ll \frac{H_{2}}{p} \sum_{x_{1} x_{2} y_{1} y_{2} z=d} \frac{1}{x_{1} x_{2} y_{2}} \\
& \times \sum_{1 \leq\left|m_{1}\right| \leq p x_{1} / 2}\left|\sum_{q \sim Q / z} \sum_{\substack{s_{1}: P / y_{1} \\
\left(s_{1}, p x_{1}\right)=1}} e\left(\frac{m_{1}\left(\overline{\left.s_{1} y_{1} z h_{1}+q\right)}\right.}{x_{1} p}\right)\right| .
\end{aligned}
$$

Now for $\left|m_{1}\right| \leq p x_{1} / 2$ we have

$$
\left|\sum_{q \sim Q / z} e\left(\frac{m_{1} q}{x_{1} p}\right)\right| \leq \min \left(\frac{Q}{z}, \frac{x_{1} p}{\left|m_{1}\right|}\right)
$$

(note that $q$ now runs over all integers in its range), so the exponential sum above is no more than

$$
\begin{aligned}
& \frac{H_{2}}{p} \sum_{x_{1} x_{2} y_{1} y_{2} z=d} \frac{1}{x_{1} x_{2} y_{2}} \\
& \times \sum_{1 \leq\left|m_{1}\right| \leq p x_{1} / 2} \min \left(\frac{Q}{z}, \frac{x_{1} p}{\left|m_{1}\right|}\right)\left|\sum_{\substack{s_{1}: P / y_{1} \\
\left(s_{1}, P x_{1}\right)=1}} e\left(\frac{m_{1} \overline{s_{1} y_{1} z h_{1}}}{x_{1} p}\right)\right| .
\end{aligned}
$$

We may now apply Lemma 4 to the inner sum above giving a factor $\left(x_{1} p\right)^{1 / 2+\varepsilon}$. We then sum over $m_{1}$ either directly, for $\left|m_{1}\right| \leq p x_{1} z / q$, or in blocks for $\left|m_{1}\right|>p x_{1} z / q$ thus obtaining a bound for the above of

$$
H_{2} \sum_{x_{1} x_{2} y_{1} y_{2} z=d} \frac{x_{1}^{1 / 2+\varepsilon}}{x_{2} y_{2} z} p^{1 / 2+\varepsilon}+H_{2} \sum_{x_{1} x_{2} y_{1} y_{2} z=d} \frac{x_{1}^{1 / 2+\varepsilon}}{x_{2} y_{2}} \log (P / z) p^{1 / 2+\varepsilon} .
$$

Combined these are

$$
\ll H_{2} p^{1 / 2+\varepsilon} d^{1 / 2+\varepsilon} .
$$

The remaining part of the multiple exponential sum in (3.9) is

$$
\sum_{x_{1} x_{2} y_{1} y_{2} z=d} \frac{1}{p^{2} x_{1} x_{2}} \sum_{\substack{1 \leq\left|m_{1}\right| \leq p x_{1} / 2 \\ 1 \leq\left|m_{2}\right| \leq p x_{2} / 2}} \sum_{q \sim Q / z} \sum_{\substack{s_{1}: P / y_{1} \\\left(s_{1}, p x_{1}\right)=1}} \sum_{\substack{s_{2}: P / y_{2} \\ 0<\left|h_{2}\right| \leq H_{2}}} S,
$$

where

$$
S=e\left(\frac{m_{1}\left(\overline{s_{1} y_{1} z} h_{1}+q\right)}{x_{1} p}\right) e\left(\frac{m_{2}\left(s_{2} y_{2} z q+h_{2}\right)}{x_{2} p}\right) .
$$


This has order of magnitude no greater than

$$
\begin{aligned}
\sum_{x_{1} \ldots z} \frac{1}{p^{2} x_{1} x_{2}} \sum_{\substack{m_{1}, m_{2} \\
q \sim Q / z}} \min \left(H_{2}, \frac{x_{2} p}{\left|m_{2}\right|}\right) \\
\times\left|\sum_{\substack{s_{1}: P / y_{1} \\
\left(s_{1}, p x_{1}\right)=1}} e\left(\frac{m_{1}\left(\overline{\left.s_{1} y_{1} z h_{1}+q\right)}\right.}{x_{1} p}\right)\right|\left|\sum_{s_{2}: P / y_{2}} e\left(\frac{m_{2} s_{2} y_{2} z q}{x_{2} p}\right)\right| ;
\end{aligned}
$$

here we have summed over $h_{2}$. Next we sum over $s_{2}$ to give a bound of

$$
\sum_{x_{1} \ldots z} \frac{1}{p^{2} x_{1} x_{2}} \sum_{\substack{m_{2} \\ q \sim Q / z}} \min \left(H_{2}, \frac{x_{2} p}{\left|m_{2}\right|}\right) \min \left(\frac{P}{y_{2}}, \frac{1}{\left\|\frac{m_{2} y_{2} z q}{x_{2} p}\right\|}\right) T,
$$

where

$$
T=\sum_{1 \leq\left|m_{1}\right| \leq p x_{1} / 2}\left|\sum_{\substack{s_{1}: P / y_{1} \\\left(s_{1}, p x_{1}\right)=1}} e\left(\frac{m_{1}\left(\overline{s_{1} y_{1} z} h_{1}+q\right)}{x_{1} p}\right)\right| .
$$

We can now apply Lemma 4 directly to $T$. For the sum over $m_{2}$ and $q$ we consider the parts where $x_{2} p$ does and does not divide $m_{2} y_{2} z q$ separately. In the first case we combine the variables $q$ and $m_{2}$ to give a single variable running over a large range. This will also produce a $Q^{\varepsilon}$ term as an upper bound on the number of divisors of the variable. This part of the sum then is

$$
\ll \sum_{x_{1} \ldots z} \frac{1}{p^{2} x_{1} x_{2}}\left(x_{2} p\right) \log \left(x_{2} p\right)\left(\frac{Q}{z x_{2} p}\right) x_{2} p \log \left(x_{2} p\right) Q^{\varepsilon}\left(x_{1} p\right)\left(x_{1} p\right)^{1 / 2+\varepsilon / 2},
$$

which is

$$
\ll P^{1 / 2+\varepsilon} Q^{1+\varepsilon} \sum_{x_{1} x_{2} y_{1} y_{2} z=d} x_{1}^{1 / 2+\varepsilon / 2} .
$$

This gives a bound of $P^{1 / 2+\varepsilon} Q^{1+\varepsilon} d^{1 / 2+\varepsilon}$, and since $H_{2} \gg Q P^{-\delta}$, this is

$$
\ll H_{2} P^{1 / 2+\varepsilon} Q^{\varepsilon} d^{1 / 2+\varepsilon} \text {. }
$$

When $x_{2} p$ divides $m_{2} y_{2} z q$ we are forced to take the trivial bound $P / y_{2}$ on the sum over $s_{2}$ but since this occurs for a small range of $m_{2}$ values the bound we get is less than the bound given above.

By (3.10)-(3.12) the bound for the remainder term $R_{d}$ as expressed in $(3.9)$ is

$$
\ll \frac{H_{2} Q^{1+\varepsilon} d^{1 / 2+\varepsilon}}{p^{1 / 2}} .
$$

Hence

$$
\sum_{\substack{d \leq Y \\(d, h)=1}} \mu^{2}(d) d^{\varepsilon}\left|R_{d}\right| \ll H_{2} Q^{1+\varepsilon} P^{-1 / 2} Y^{3 / 2+2 \varepsilon} .
$$


By taking $Y=P^{1 / 4}$ and both occurrences of $\varepsilon$ as $1 / 100$ we can apply Lemma $3 \mathrm{~b}$ to state that for fixed $h_{1}$ and $p$, the number of solutions to the inequalities (3.1) is

$$
\leq \frac{\Delta H_{2} \theta^{3} Q}{\log ^{5} P}\left(\frac{h_{1}}{\phi\left(h_{1}\right)}\right)^{5}+O\left(H_{2} Q^{63 / 64}\right) .
$$

Now

$$
\sum_{1 \leq h_{1} \leq H_{1}}\left(\frac{h_{1}}{\phi\left(h_{1}\right)}\right)^{5}<\Delta^{\prime} H_{1}
$$

so summing (3.13) over $p$ and $h_{1}$ gives the bound

$$
\frac{\Delta H_{1} H_{2} \theta^{4} P Q}{(\log P)^{3}(\log Q)^{3}}+O\left(H_{1} H_{2} P Q^{63 / 64}\right) .
$$

Here we used the fact that $4 \log P \geq \log Q$. This completes the third and final case of the proof of Lemma 2 .

We should now mention some details regarding the proof of Lemma 2 for $k>2$. The proof still falls into the three cases used when $k=2$. If we look at these in turn, Case 1 generalises instantly and the difference is merely one of notation. Case 2 is where we have $P$ close to $Q$ and with none of the $\psi_{i}(P)$ functions being "small". This case too generalises in a straightforward way. The sieve result used is again Lemma 3a, applied with $\kappa=2 k$. Result 3 is employed, giving us the equation

$$
\sum_{n \in \mathcal{A}_{d}} 1=\sum_{x_{1} \ldots x_{k} y_{1} \ldots y_{k}=d}\left(\sum_{r_{1}, \ldots, r_{k}, p}\left(\frac{2^{k} H_{1} \ldots H_{k}}{y_{1} \ldots y_{k} q^{k}}+T_{1}+\ldots+T_{k}\right)+E\right),
$$

where the $T_{j}$ terms are of the form

$$
T_{j}=(2 j-1) \frac{\theta_{j}}{(L+1)^{j}} \sum_{\substack{1 \leq i_{a} \leq k \\ i_{b} \neq i_{c}}} \frac{2 H_{i_{1}}}{q y_{i_{1}}} \times \ldots \times \frac{2 H_{i_{k-j}}}{q y_{i_{k-j}}} .
$$

It should be noted that the parameter $L$ can be set to $Q$ as before. The exponential sum $E$ looks like

$$
\sum_{x_{1} \ldots x_{k} y_{1} \ldots y_{k}=d} \sum_{r_{1}, \ldots, r_{k}, p} c(\mathbf{m})\left|\sum_{r_{1}, \ldots, r_{k}, p} e\left(\frac{m_{1} r_{1} x_{1} p}{y_{1} q}+\ldots+\frac{m_{k} r_{k} x_{k} p}{y_{k} q}\right)\right| .
$$

Since this is symmetrical in the $k$ variables, the generalisation from $k=2$ is immediate. In Case 3 of the proof one might expect complications to arise from the fact that we have more than two $\psi(p)$ functions of different sizes. However, for each $k$ only one of the functions can be small in the sense that

$$
\psi(p) \ll p^{\delta-1} \quad \text { for any } \delta>0,
$$


since if more than one of these functions had this property then our sum (1.2) would converge. It may be the case that the product of $l$ of the functions is small in the sense given above thus forcing the product of the remaining $k-l$ functions to be large in the sense that

$$
\psi_{l+1}(p) \ldots \psi_{k}(p) \gg p^{-\delta} \quad \text { for any } \delta>0,
$$

but no problems arise from functions being large in this sense. For Case 3 then we employ a $(2 k+1)$-dimensional sieve with $\mathcal{A}$ equal to

$$
\left\{r_{1} \ldots r_{k} s_{1} \ldots s_{k} q\left|r_{1} p-s_{1} q=h_{1},\right| r_{i} p-s_{i} q \mid<H_{i}, s_{i}: P \text { and } q \sim Q\right\} .
$$

The remainder terms in the sieve are dealt with using exponential sums as for $k=2$ but over $k$ variables this time, $m_{1} \ldots m_{k}$. The part of the sum having all $m_{i}$ zero except $m_{1}$ is dealt with using the Kloosterman sum estimate as before.

4. Additional proofs. The proof of Result 2 is as follows.

Firstly we need the following lemma which, given a measurable subset $A$ of $\mathbb{R}^{k}$, provides a useful sufficient condition for $\mathbb{R}^{k} \backslash A$ to be a null set.

Lemma 5. Let $A$ be as above and suppose that for every open cube $C \subseteq \mathbb{R}^{k}$ we have

$$
\lambda^{k}(A \cap C) \geq \delta \lambda^{k}(C)
$$

where $\delta>0$ is a constant independent of the choice of $C$. Then $\lambda^{k}\left(\mathbb{R}^{k} \backslash A\right)$ $=0$.

Proof. Corollary 6.2.6 on page 184 of [1] states that, if $S$ is a (Lebesgue) measurable subset of $\mathbb{R}^{k}$, then almost all points of $S$ are density points of $S$. Applying this to the set $A^{\mathrm{c}}$, the complement of $A$ relative to $\mathbb{R}^{k}$, and assuming for a contradiction that $\lambda^{k}\left(A^{\mathrm{c}}\right) \neq 0$, we get

$$
\begin{aligned}
& \exists x \in A^{\mathrm{c}} \forall \varepsilon>0 \exists \delta^{\prime}>0 \\
& \qquad\left|\frac{\lambda^{k}\left(A^{\mathrm{c}} \cap C\right)}{\lambda^{k}(C)}-1\right|<\varepsilon \quad \text { for all cubes } C \text { with } e(C)<\delta^{\prime} .
\end{aligned}
$$

(Here $e(C)$ denotes the edge length of $C$.) Applying the above with $\varepsilon=\delta / 2$ (where $\delta$ is as in the lemma) and letting $C$ be an open cube with $e(C)<\delta_{\varepsilon}^{\prime}$ we have:

$$
\left|\frac{\lambda^{k}\left(A^{\mathrm{c}} \cap C\right)}{\lambda^{k}(C)}-1\right|<\frac{\delta}{2}
$$

hence $1-\lambda^{k}\left(A^{\mathrm{c}} \cap C\right) / \lambda^{k}(C)<\delta / 2$ and so $\lambda^{k}\left(A^{\mathrm{c}} \cap C\right)>(1-\delta / 2) \lambda^{k}(C)$. 
But

$$
\begin{aligned}
\lambda^{k}(C) & =\lambda^{k}\left(A^{\mathrm{c}} \cap C\right)+\lambda^{k}(A \cap C) \\
& >(1-\delta / 2) \lambda^{k}(C)+\delta \lambda^{k}(C) \quad \text { (by assumption in the lemma) } \\
& =(1+\delta / 2) \lambda^{k}(C) \quad(\text { contradiction, since } \delta>0) .
\end{aligned}
$$

Hence we must have $\lambda^{k}\left(A^{\mathrm{c}}\right)=0$, which proves the lemma.

The result will now follow from two lemmas which allow us to apply Lemma 5 with $A=V^{\mathrm{c}} \cup \mathcal{D}$.

Lemma 6. Let $C$ be an open cube with $C \subseteq V$ and let $B_{n}=C \cap D_{n}$. Then

$$
\lambda^{k}(\mathcal{D} \cap C) \geq \delta \lambda^{k}(C) .
$$

Proof. Firstly, we apply Lemma 5 on p. 17 of [8] to the sequence of measurable sets $\left(B_{n}\right)$ and get

$$
\lambda^{k}(\mathcal{B}) \geq \lim _{N \rightarrow \infty}\left(\sum_{n=1}^{\infty} \lambda^{k}\left(B_{n}\right)\right)^{2}\left(\sum_{1 \leq m, n \leq N} \lambda^{k}\left(B_{m} \cap B_{n}\right)\right)^{-1},
$$

where $\mathcal{B}=C \cap \mathcal{D}$. The lemma requires a finite measure space but since the sets $\left(B_{n}\right)$ all lie in the finite cube $C$ this is satisfied. The result then follows from (4.1) and (1.4) of Result 2.

Lemma 7. Let $A=V^{\mathrm{c}} \cup \mathcal{D}$ and $C$ be an open cube in $\mathbb{R}^{k}$. Then

$$
\lambda^{k}(A \cap C) \geq \delta \lambda^{k}(C)
$$

where $\delta>0$ is a constant independent of the choice of $C$.

Proof. Let $G=C \cap V$; then $G$ is an open, measurable subset of $\mathbb{R}^{k}$. Hence Lemma 1.4.2 (p. 28) of [1] ensures we can say

$$
G=\bigcup_{i=1}^{\infty} C_{i} \quad \text { where the } C_{i} \text { are disjoint half-open cubes. }
$$

We have

$$
\begin{aligned}
(*) \quad \lambda^{k}(\mathcal{D} \cap G) & =\lambda^{k}\left(\mathcal{D} \cap \bigcup_{i=1}^{\infty} C_{i}\right)=\lambda^{k}\left(\bigcup_{i=1}^{\infty}\left(\mathcal{D} \cap C_{i}\right)\right) \quad\left(C_{i} \text { disjoint }\right) \\
& =\sum_{i=1}^{\infty} \lambda^{k}\left(\mathcal{D} \cap C_{i}\right)=\sum_{i=1}^{\infty} \lambda^{k}\left(\mathcal{D} \cap C_{i}^{\circ}\right) \\
& \geq \sum_{i=1}^{\infty} \delta \lambda^{k}\left(C_{i}^{\circ}\right) \quad(\text { by Lemma } 6) \\
& =\sum_{i=1}^{\infty} \delta \lambda^{k}\left(C_{i}\right)=\delta \lambda^{k}\left(\bigcup_{i=1}^{\infty} C_{i}\right)=\delta \lambda^{k}(G) .
\end{aligned}
$$


Hence,

$$
\begin{aligned}
\lambda^{k}(A \cap C) & =\lambda^{k}\left(\left(V^{\mathrm{c}} \cup \mathcal{D}\right) \cap C\right) & & \\
& =\lambda^{k}\left(V^{\mathrm{c}} \cap C\right)+\lambda^{k}(\mathcal{D} \cap C) & & (\text { since } \mathcal{D} \subseteq V) \\
& =\lambda^{k}(C \backslash V)+\lambda^{k}(\mathcal{D} \cap(C \cap V)) & & (\text { since } \mathcal{D} \subseteq V) \\
& =\lambda^{k}(C \backslash V)+\lambda^{k}(\mathcal{D} \cap G) & & \\
& \geq \lambda^{k}(C \backslash V)+\delta \lambda^{k}(G) & & \text { (by }(*)) \\
& \geq \delta \lambda^{k}(C \backslash V)+\delta \lambda^{k}(C \cap V) & & \text { (letting } \delta<1) \\
& =\delta \lambda^{k}(C) & & \text { (as required). }
\end{aligned}
$$

We have shown that $A=V^{\mathrm{c}} \cup \mathcal{D}$ fulfils the criteria of Lemma 5 so we have $\lambda^{k}\left(\mathbb{R}^{k} \backslash\left(V^{\mathrm{c}} \cup \mathcal{D}\right)\right)=0$; but this implies that $\lambda^{k}\left(V \cap\left(\mathbb{R}^{k} \backslash \mathcal{D}\right)\right)=0$ because $\mathcal{D} \subseteq V \subseteq \mathbb{R}^{k}$ and so $\lambda^{k}(V \backslash \mathcal{D})=0$ as required.

Acknowledgements. This work was undertaken while the author was in receipt of a Cardiff University studentship. The author would like to thank Prof. Glyn Harman for his encouragement and good advice.

\section{References}

[1] D. L. Cohn, Measure Theory, Birkhäuser, Boston, 1980.

[2] R. J. Duffin and A. C. Schaffer, Khinchine's problem in metric Diophantine approximation, Duke Math. J. 8 (1941), 243-255.

[3] H. Halberstam and H.-R. Richert, Sieve Methods, Academic Press, New York, 1974.

[4] G. Harman, Metric Number Theory, Clarendon Press, Oxford, 1998.

[5] - Metric Diophantine approximation with two restricted variables III. Two prime numbers, J. Number Theory 29 (1988), 364-375.

[6] C. Hooley, Applications of Sieve Methods, Cambridge, 1976.

[7] A. Ya. Khinchin, Continued Fractions, English translation of third edition by Univ. of Chicago Press, 1964.

[8] V. G. Sprindžuk, Metric Theory of Diophantine Approximations, Winston and Wiley, Washington, 1979.

School of Mathematics

Cardiff University

Senghennydd Road

PO Box 926

Cardiff CF2 4HY, Wales, U.K.

E-mail: JonesHR@cardiff.ac.uk

HuwJ@zuken.co.uk

Received on 26.10.1998

and in revised form on 27.12.1999 